\title{
SOME CHARACTERIZATIONS OF SPECIALLY MULTIPLICATIVE FUNCTIONS
}

\author{
PENTTI HAUKKANEN
}

Received 10 January 2003

\begin{abstract}
A multiplicative function $f$ is said to be specially multiplicative if there is a completely multiplicative function $f_{A}$ such that $f(m) f(n)=\sum_{d \mid(m, n)} f\left(m n / d^{2}\right) f_{A}(d)$ for all $m$ and $n$. For example, the divisor functions and Ramanujan's $\tau$-function are specially multiplicative functions. Some characterizations of specially multiplicative functions are given in the literature. In this paper, we provide some further characterizations of specially multiplicative functions.
\end{abstract}

2000 Mathematics Subject Classification: 11A25.

1. Introduction. An arithmetical function $f$ is said to be multiplicative if $f(1)=1$ and

$$
f(m) f(n)=f(m n)
$$

whenever $(m, n)=1$. If (1.1) holds for all $m$ and $n$, then $f$ is said to be completely multiplicative. A multiplicative function is known if the values $f\left(p^{n}\right)$ are known for all prime numbers $p$ and positive integers $n$. A completely multiplicative function is known if the values $f(p)$ are known for all prime numbers $p$.

A multiplicative function $f$ is said to be specially multiplicative if there is a completely multiplicative function $f_{A}$ such that

$$
f(m) f(n)=\sum_{d \mid(m, n)} f\left(\frac{m n}{d^{2}}\right) f_{A}(d)
$$

for all $m$ and $n$, or equivalently

$$
f(m n)=\sum_{d \mid(m, n)} f\left(\frac{m}{d}\right) f\left(\frac{n}{d}\right) \mu(d) f_{A}(d)
$$

for all $m$ and $n$, where $\mu$ is the Möbius function. If $f_{A}=\delta$, where $\delta(1)=1$ and $\delta(n)=0$ for $n>1$, then (1.2) reduces to (1.1). Therefore, the class of completely multiplicative functions is a subclass of the class of specially multiplicative functions. 
The study of specially multiplicative functions was initiated in [7], and arose in an effort to understand the identity

$$
\sigma_{\alpha}(m n)=\sum_{d \mid(m, n)} \sigma_{\alpha}\left(\frac{m}{d}\right) \sigma_{\alpha}\left(\frac{n}{d}\right) \mu(d) d^{\alpha},
$$

where $\sigma_{\alpha}(n)$ denotes the sum of the $\alpha$ th powers of the positive divisors of $n$. Vaidyanathaswamy used the term "quadratic function," while the term "specially multiplicative function" was coined by Lehmer [3]. For more background information, reference is made to the books by McCarthy [4] and Sivaramakrishnan [6].

The Dirichlet convolution of two arithmetical functions $f$ and $g$ is defined as

$$
(f * g)(n)=\sum_{d \mid n} f(d) g\left(\frac{n}{d}\right)
$$

The function $\delta$ serves as the identity under the Dirichlet convolution. An arithmetical function $f$ possesses a Dirichlet inverse $f^{-1}$ if and only if $f(1) \neq 0$.

We next review some basic characterizations of specially multiplicative functions, see $[4,6]$.

Proposition 1.1. The following statements are equivalent.

(1) The function $f$ is a specially multiplicative function.

(2) The function $f$ is the Dirichlet convolution of two completely multiplicative functions $a$ and $b$. (In this case $f_{A}=a b$, the usual product of $a$ and b.)

(3) The function $f$ is a multiplicative function, and for each prime number $p$,

$$
f^{-1}\left(p^{n}\right)=0, \quad n \geq 3
$$

(In this case $f_{A}(p)=f^{-1}\left(p^{2}\right)$ for all prime numbers $p$.)

(4) The function $f$ is a multiplicative function, and for each prime number $p$, there exists a complex number $g(p)$ such that

$$
f\left(p^{n+1}\right)=f(p) f\left(p^{n}\right)-g(p) f\left(p^{n-1}\right), \quad n \geq 1 .
$$

(In this case $f_{A}(p)=g(p)$ for all prime numbers $p$.)

(5) The function $f$ is a multiplicative function, and for each prime number $p$, there exists a complex number $g(p)$ such that

$$
f\left(p^{n}\right)=\sum_{k=0}^{\lfloor n / 2\rfloor}(-1)^{k}\left(\begin{array}{c}
n-k \\
k
\end{array}\right)[f(p)]^{n-2 k}[g(p)]^{k}, \quad n \geq 0 .
$$

(In this case $f_{A}(p)=g(p)$ for all prime numbers $p$.) 
REMARK 1.2. Completely multiplicative functions $a$ and $b$ in part 2 need not be unique. The usual product $a b$, however, is unique. For example, let $a, b, c$, and $d$ be completely multiplicative functions such that $a(p)=1$ and $b(p)=2$ for all prime numbers $p$, and $c(2)=2, c(p)=1, d(2)=1$, and $d(p)=2$ for all prime numbers $p \neq 2$. Then $a * b=c * d$, but $a, b \neq c$ and $a, b \neq d$. However, $a b=c d$.

The purpose of this paper is to provide some further characterizations of specially multiplicative functions. As applications, we obtain formulas for the usual products $\sigma_{\alpha} \phi_{\beta}, \sigma_{\alpha} \sigma_{\beta}$, and $\sigma_{\alpha} \tau$, where $\phi_{\beta}$ is a generalized Euler totient function and $\tau$ is Ramanujan's $\tau$-function. The function $\phi_{\beta}$ is given by $\phi_{\beta}=$ $N^{\beta} * \mu$, where $N^{\beta}(n)=n^{\beta}$ for all $n$. In particular, we denote $N^{1}=N, N^{0}=\zeta$, and $\phi_{1}=\phi$, where $\phi$ is the Euler totient function. Ramanujan's $\tau$-function is a specially multiplicative function with $\tau_{A}=N^{11}$.

In the characterizations, we need the concepts of the unitary convolution and the $k$ th convolute. The unitary convolution of two arithmetical functions $f$ and $g$ is defined as

$$
(f \oplus g)(n)=\sum_{d \| n} f(d) g\left(\frac{n}{d}\right)
$$

where $d \| n$ means that $d \mid n,(d, n / d)=1$. The $k$ th convolute of an arithmetical function $f$ is defined as $\Omega_{k}(f)(n)=f\left(n^{1 / k}\right)$ if $n$ is a $k$ th power, and $\Omega_{k}(f)(n)=0$ otherwise.

\section{Characterizations}

THEOREM 2.1. If $f$ is a specially multiplicative function and $g$ is a completely multiplicative function, then

$$
h * f(g * \mu)=f g,
$$

where $h$ is the specially multiplicative function such that

$$
h(p)=f(p), \quad h_{A}(p)=g(p) f_{A}(p)
$$

for all prime numbers $p$. Conversely, if $f(1)=1$ and there exist completely multiplicative functions $a, b, g$, and $k$ such that

$$
a * b * f(g * \mu)=f g,
$$

where

$$
a(p)+b(p)=f(p), \quad a(p) b(p)=g(p) k(p), \quad(g * \mu)(n) \neq g(n)
$$

for all prime numbers $p$ and integers $n(\geq 2)$, then $f$ is a specially multiplicative function with $f_{A}=k$. 
Proof. By multiplicativity, it suffices to show that (2.1) holds at prime powers, that is,

$$
[f(g * \mu)]\left(p^{e}\right)=\left(f g * h^{-1}\right)\left(p^{e}\right)
$$

for all prime powers $p^{e}$. If $e=1$, then both sides of (2.5) are equal to $f(p) g(p)-$ $f(p)$. Assume that $e \geq 2$. Then

$$
\begin{aligned}
\left(f g * h^{-1}\right)\left(p^{e}\right)= & f\left(p^{e}\right) g\left(p^{e}\right)+f\left(p^{e-1}\right) g\left(p^{e-1}\right) h^{-1}(p) \\
& +f\left(p^{e-2}\right) g\left(p^{e-2}\right) h^{-1}\left(p^{2}\right) \\
= & f\left(p^{e}\right) g\left(p^{e}\right)-f\left(p^{e-1}\right) g\left(p^{e-1}\right) f(p) \\
& +f\left(p^{e-2}\right) g\left(p^{e-2}\right) g(p) f_{A}(p) .
\end{aligned}
$$

By (1.7), we obtain

$$
\left(f g * h^{-1}\right)\left(p^{e}\right)=f\left(p^{e}\right) g\left(p^{e}\right)-f\left(p^{e}\right) g\left(p^{e-1}\right)=f\left(p^{e}\right)(g * \mu)\left(p^{e}\right) .
$$

Thus we have proved (2.5).

To prove the converse, we write (2.3) in the form

$$
(f(g * \mu))(n)=\left(f g * a^{-1} * b^{-1}\right)(n) .
$$

We write $n=p^{e+1}(e \geq 1)$ and, after some simplifications, obtain

$$
f\left(p^{e+1}\right)=f\left(p^{e}\right) f(p)-f\left(p^{e-1}\right) k(p) .
$$

Therefore, by (1.7), it remains to prove that $f$ is multiplicative. Denote $n=$ $p_{1}^{e_{1}} \cdots p_{r}^{e_{r}} p_{r+1} \cdots p_{r+s}$, where $e_{i}>1(i=1,2, \ldots, r)$. We proceed by induction on $e_{1}+\cdots+e_{r}+s$ to prove that

$$
f(n)=f\left(p_{1}^{e_{1}}\right) \cdots f\left(p_{r}^{e_{r}}\right) f\left(p_{r+1}\right) \cdots f\left(p_{r+s}\right) .
$$

If $e_{1}+\cdots+e_{r}+s=1$, then (2.10) holds. Suppose that (2.10) holds when $e_{1}+$ $\cdots+e_{r}+s<m$. Then for $e_{1}+\cdots+e_{r}+s=m$, we have after some manipulation

$$
\begin{aligned}
f(n) & (g * \mu)(n) \\
= & \left(f g * a^{-1} * b^{-1}\right)(n) \\
= & f(n) g(n)+\sum_{\substack{d \mid n \\
d>1}} f\left(\frac{n}{d}\right) g\left(\frac{n}{d}\right)\left(a^{-1} * b^{-1}\right)(d) \\
= & f(n) g(n)-\prod_{p^{e} \| n} f\left(p^{e}\right) g\left(p^{e}\right)+\prod_{p^{e} \| n}\left(f g * a^{-1} * b^{-1}\right)\left(p^{e}\right)
\end{aligned}
$$




$$
\begin{aligned}
= & f(n) g(n)-\prod_{p^{e} \| n} f\left(p^{e}\right) g\left(p^{e}\right) \\
+ & \prod_{i=1}^{r}\left[f\left(p_{i}^{e_{i}}\right) g\left(p_{i}^{e_{i}}\right)-f\left(p_{i}^{e_{i}-1}\right) f\left(p_{i}\right) g\left(p_{i}^{e_{i}-1}\right)+f\left(p_{i}^{e_{i}-2}\right) k\left(p_{i}\right) g\left(p_{i}^{e_{i}-1}\right)\right] \\
& \times \prod_{i=1}^{s}\left(f\left(p_{r+i}\right) g\left(p_{r+i}\right)-f\left(p_{r+i}\right)\right) .
\end{aligned}
$$

Using (2.9), we obtain

$$
f(n)(g * \mu)(n)=f(n) g(n)-g(n) \prod_{p^{e} \| n} f\left(p^{e}\right)+(g * \mu)(n) \prod_{p^{e} \| n} f\left(p^{e}\right) .
$$

This gives (2.10).

REMARK 2.2. The converse part of Theorem 2.1 can also be written as follows. If $f(1)=1$ and there exist completely multiplicative functions $g$ and $k$, and a specially multiplicative function $h$ such that

$$
h * f(g * \mu)=f g,
$$

where

$$
h(p)=f(p), \quad h_{A}(p)=g(p) k(p), \quad(g * \mu)(n) \neq g(n)
$$

for all prime numbers $p$ and integers $n(\geq 2)$, then $f$ is a specially multiplicative function with $f_{A}=k$.

COROLLARY 2.3. If $f$ is a specially multiplicative function, then

$$
h * f \phi=f N,
$$

where $h$ is the specially multiplicative function such that

$$
h(p)=f(p), \quad h_{A}(p)=p f_{A}(p)
$$

for all prime numbers $p$. Conversely, if $f(1)=1$ and if there exist completely multiplicative functions $a, b$, and $k$ such that

$$
a * b * f \phi=f N
$$

where

$$
a(p)+b(p)=f(p), \quad a(p) b(p)=p k(p)
$$

for all prime numbers $p$, then $f$ is a specially multiplicative function with $f_{A}=k$. 
COROLLARY 2.4. If $f$ and $g$ are completely multiplicative functions, then

$$
f * f(g * \mu)=f g .
$$

Conversely, if $f(1)=1$ and if there exists a completely multiplicative function $g$ such that

$$
f * f(g * \mu)=f g,
$$

where

$$
(g * \mu)(n) \neq g(n)
$$

for all integers $n(\geq 2)$, then $f$ is a completely multiplicative function.

COROLLARY 2.5 (Sivaramakrishnan [5]). If $f(1)=1$, then $f$ is a completely multiplicative function if and only if

$$
f * f \phi=f N .
$$

EXAMPLE 2.6. We have

$$
\sigma_{\alpha} \phi_{\beta}=\sigma_{\alpha} N^{\beta} * h^{-1},
$$

where $h$ is the specially multiplicative function such that

$$
h(p)=\sigma_{\alpha}(p)=p^{\alpha}+1, \quad h_{A}(p)=p^{\beta} p^{\alpha}=p^{\alpha+\beta}
$$

for all prime numbers $p$.

THEOREM 2.7. If $f$ is a specially multiplicative function and $g$ is a completely multiplicative function, then

$$
f(g * \mu)=f g *\left(\mu f \oplus \Omega_{2}\left(\mu^{2} f_{A} g\right)\right) .
$$

Conversely, if $f(1) \neq 0$ and if there exist completely multiplicative functions $g$ and $k$ such that

$$
f(g * \mu)=f g *\left(\mu f \oplus \Omega_{2}\left(\mu^{2} k g\right)\right),
$$

where

$$
(g * \mu)(n) \neq g(n)
$$

for all $n$, then $f$ is a specially multiplicative function with $f_{A}=k$.

Proof. We observe that

$$
\begin{aligned}
\left(\mu f \oplus \Omega_{2}\left(\mu^{2} f_{A} g\right)\right)(p) & =-f(p), \\
\left(\mu f \oplus \Omega_{2}\left(\mu^{2} f_{A} g\right)\right)\left(p^{2}\right) & =f_{A}(p) g(p), \\
\left(\mu f \oplus \Omega_{2}\left(\mu^{2} f_{A} g\right)\right)\left(p^{n}\right) & =0
\end{aligned}
$$


for all prime numbers $p$ and integers $n(\geq 3)$. Therefore $\mu f \oplus \Omega_{2}\left(\mu^{2} f_{A} g\right)=h^{-1}$, where $h$ is the specially multiplicative function in Theorem 2.1. Thus (2.25) follows from (2.1).

The converse follows from Theorem 2.1 since $\mu f \oplus \Omega_{2}\left(\mu^{2} g k\right)=a^{-1} * b^{-1}$, where $a$ and $b$ are completely multiplicative functions as given in Theorem 2.1.

THEOREM 2.8. If $f$ is a specially multiplicative function and $g$ is a completely multiplicative function, then

$$
f(g * \mu)=f g *\left(f^{-1} \oplus \Omega_{2}\left(\mu^{2} f_{A}(g \oplus \mu)\right)\right) .
$$

Conversely, if $f(1)=1$ and there exist completely multiplicative functions $c, d$, and $g$ such that

$$
f(g * \mu)=f g *\left((c * d)^{-1} \oplus \Omega_{2}\left(\mu^{2} c d(g \oplus \mu)\right)\right),
$$

where

$$
c(p)+d(p)=f(p), \quad(g * \mu)(n) \neq g(n)
$$

for all prime numbers $p$ and integers $n(\geq 2)$, then $f$ is the specially multiplicative function given as $f=c * d$.

Proof. Proof of Theorem 2.8 is similar to that of Theorem 2.7.

EXAMPLE 2.9. We have

$$
\begin{aligned}
& \sigma_{\alpha} \phi_{\beta}=\sigma_{\alpha} N^{\beta} *\left(\mu \sigma_{\alpha} \oplus \Omega_{2}\left(\mu^{2} N^{\alpha+\beta}\right)\right), \\
& \sigma_{\alpha} \phi_{\beta}=\sigma_{\alpha} N^{\beta} *\left(\sigma_{\alpha}^{-1} \oplus \Omega_{2}\left(\mu^{2} N^{\alpha}\left(N^{\beta} \oplus \mu\right)\right)\right) .
\end{aligned}
$$

LEMMA 2.10. Suppose that $f$ is an arithmetical function such that $f(1)=1$ and $f^{-1}\left(p^{i}\right)=0$ for $3 \leq i<k(k \geq 4)$. Then

$$
f\left(p^{k}\right)=f(p) f\left(p^{k-1}\right)-f^{-1}\left(p^{2}\right) f\left(p^{k-2}\right)-f^{-1}\left(p^{k}\right) .
$$

Proof. Lemma 2.10 follows from the equation

$$
\sum_{i=0}^{k} f^{-1}\left(p^{i}\right) f\left(p^{k-i}\right)=0 .
$$

THEOREM 2.11. If $f$ is a specially multiplicative function and $g$ is a completely multiplicative function, then

$$
f(g * \zeta)=f g * f * \Omega_{2}\left(f_{A} g\right)^{-1} .
$$


Conversely, if $f$ is a multiplicative function such that

$$
f(g * \zeta)=f g * f * \Omega_{2}(h g)^{-1}
$$

where $g$ is a completely multiplicative function with $g(p)(g * \zeta)\left(p^{e}\right) \neq 0$ for all prime powers $p^{e}$ and where $h$ is a completely multiplicative function, then $f$ is a specially multiplicative function with $f_{A}=h$.

Proof. Let $f=a * b$, where $a$ and $b$ are completely multiplicative functions. It is known [7] that

$$
f(g * \zeta)=(a * b)(g * \zeta)=a g * a \zeta * b g * b \zeta * \Omega_{2}(a b g \zeta)^{-1}
$$

Using elementary properties of arithmetical functions, we obtain

$$
f(g * \zeta)=(a * b) g *(a * b) * \Omega_{2}\left(f_{A} g\right)^{-1}=f g * f * \Omega_{2}\left(f_{A} g\right)^{-1}
$$

This proves (2.35).

Assume that (2.36) holds. Then (2.36) at $p^{2}$ gives

$$
h(p)=f(p)^{2}-f\left(p^{2}\right)
$$

Since $f^{-1}\left(p^{2}\right)=f(p)^{2}-f\left(p^{2}\right)$ for all multiplicative functions, we obtain

$$
h(p)=f^{-1}\left(p^{2}\right)
$$

We next prove that

$$
f^{-1}\left(p^{i}\right)=0 \quad \forall i \geq 3
$$

We proceed by induction on $i$. Calculating (2.36) at $p^{3}$ and using (2.40) gives

$$
f\left(p^{3}\right)=f(p) f\left(p^{2}\right)-f(p) f^{-1}\left(p^{2}\right) .
$$

Since

$$
f\left(p^{3}\right)-f\left(p^{2}\right) f(p)+f(p) f^{-1}\left(p^{2}\right)+f^{-1}\left(p^{3}\right)=0,
$$

we see that $f^{-1}\left(p^{3}\right)=0$.

Suppose that $f^{-1}\left(p^{i}\right)=0$ for all $3 \leq i<k(k>3)$. We write (2.36) as

$$
f(g * \zeta) * f^{-1}=f g * \Omega_{2}(h g)^{-1}
$$


Suppose that $k$ is even, say $k=2 e(e>1)$. At $p^{2 e}$, the left-hand side of (2.44) becomes

$$
\begin{aligned}
\sum_{i=0}^{2 e} f\left(p^{i}\right)( & g * \zeta)\left(p^{i}\right) f^{-1}\left(p^{2 e-i}\right) \\
= & f^{-1}\left(p^{2 e}\right)+f\left(p^{2 e-2}\right)(g * \zeta)\left(p^{2 e-2}\right) f^{-1}\left(p^{2}\right) \\
& +f\left(p^{2 e-1}\right)(g * \zeta)\left(p^{2 e-1}\right) f^{-1}(p)+f\left(p^{2 e}\right)(g * \zeta)\left(p^{2 e}\right) \\
= & f^{-1}\left(p^{2 e}\right)-f^{-1}\left(p^{2 e}\right)(g * \zeta)\left(p^{2 e-2}\right) \\
& -f(p) f\left(p^{2 e-1}\right) g\left(p^{2 e-1}\right)+f\left(p^{2 e}\right) g\left(p^{2 e-1}\right)+f\left(p^{2 e}\right) g\left(p^{2 e}\right) \\
= & f^{-1}\left(p^{2 e}\right)-f^{-1}\left(p^{2 e}\right)(g * \zeta)\left(p^{2 e-1}\right) \\
& -f^{-1}\left(p^{2}\right) f\left(p^{2 e-2}\right) g\left(p^{2 e-1}\right)+f\left(p^{2 e}\right) g\left(p^{2 e}\right),
\end{aligned}
$$

where the last two equations are derived by Lemma 2.10. Further, at $p^{2 e}$, the right-hand side of (2.44) becomes

$$
\begin{aligned}
\sum_{i=0}^{2 e} f\left(p^{2 e-i}\right) & g\left(p^{2 e-i}\right) \Omega_{2}(h g)^{-1}\left(p^{i}\right) \\
= & \sum_{i=0}^{e} f\left(p^{2(e-i)}\right) g\left(p^{2(e-i)}\right) \mu\left(p^{i}\right) h\left(p^{i}\right) g\left(p^{i}\right) \\
= & f\left(p^{2 e}\right) g\left(p^{2 e}\right)-f\left(p^{2(e-1)}\right) g\left(p^{2(e-1)}\right) h(p) g(p) .
\end{aligned}
$$

Now, we see that $f^{-1}\left(p^{2 e}\right)=0$, that is, $f^{-1}\left(p^{k}\right)=0$.

If $k$ is odd, a similar argument applies. Thus (2.41) holds and therefore, by (1.6), $f$ is a specially multiplicative function with $f_{A}=h$.

COROLLARY 2.12. If $f$ is a specially multiplicative function, then

$$
f \sigma_{0}=f * f * \Omega_{2}\left(f_{A}\right)^{-1} .
$$

Conversely, if $f$ is a multiplicative function such that

$$
f \sigma_{0}=f * f * \Omega_{2}(h)^{-1},
$$

where $h$ is a completely multiplicative function, then $f$ is a specially multiplicative function with $f_{A}=h$.

COROLLARY 2.13 (Apostol [1]). If $f$ and $g$ are completely multiplicative functions, then

$$
f(g * \zeta)=f g * f
$$

Conversely, if $f$ is a multiplicative function such that

$$
f(g * \zeta)=f g * f
$$


where $g$ is a completely multiplicative function with $g(p)(g * \zeta)\left(p^{e}\right) \neq 0$ for all prime powers $p^{e}$, then $f$ is a completely multiplicative function.

COROLlary 2.14 (Carlitz [2]). Suppose that $f$ is a multiplicative function. Then $f$ is a completely multiplicative function if and only if

$$
f \sigma_{0}=f * f .
$$

COROLLARY 2.15. There exist

$$
\begin{gathered}
\tau \sigma_{\alpha}=\tau N^{\alpha} * \tau * \Omega_{2}\left(N^{\alpha+11}\right)^{-1}, \\
\sigma_{\alpha} \sigma_{\beta}=\sigma_{\alpha} N^{\beta} * \sigma_{\alpha} * \Omega_{2}\left(N^{\alpha+\beta}\right)^{-1} .
\end{gathered}
$$

\section{REFERENCES}

[1] T. M. Apostol, Some properties of completely multiplicative arithmetical functions, Amer. Math. Monthly 78 (1971), 266-271.

[2] L. Carlitz, Completely multiplicative function, Amer. Math. Monthly 78 (1971), 1140.

[3] D. H. Lehmer, Some functions of Ramanujan, Math. Student 27 (1959), 105-116.

[4] P. J. McCarthy, Introduction to Arithmetical Functions, Universitext, SpringerVerlag, New York, 1986.

[5] R. Sivaramakrishnan, Multiplicative function and its Dirichlet inverse, Amer. Math. Monthly 77 (1970), 772-773.

[6] Classical Theory of Arithmetic Functions, Monographs and Textbooks in Pure and Applied Mathematics, vol. 126, Marcel Dekker, New York, 1989.

[7] R. Vaidyanathaswamy, The theory of multiplicative arithmetic functions, Trans. Amer. Math. Soc. 33 (1931), no. 2, 579-662.

Pentti Haukkanen: Department of Mathematics, Statistics and Philosophy, University of Tampere, FIN-33014, Finland

E-mail address: Pentti . Haukkanen@uta. fi 


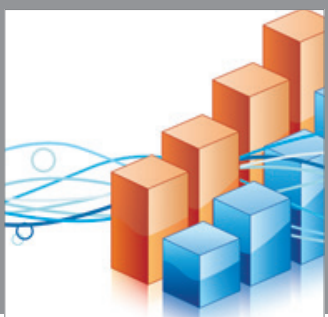

Advances in

Operations Research

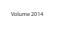

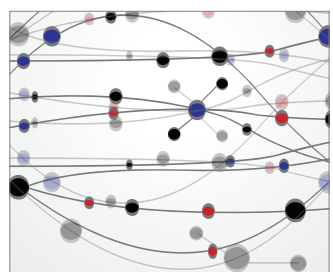

\section{The Scientific} World Journal
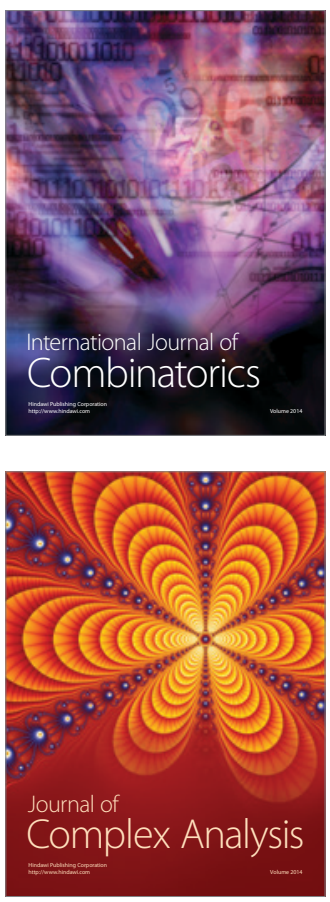

International Journal of

Mathematics and

Mathematical

Sciences
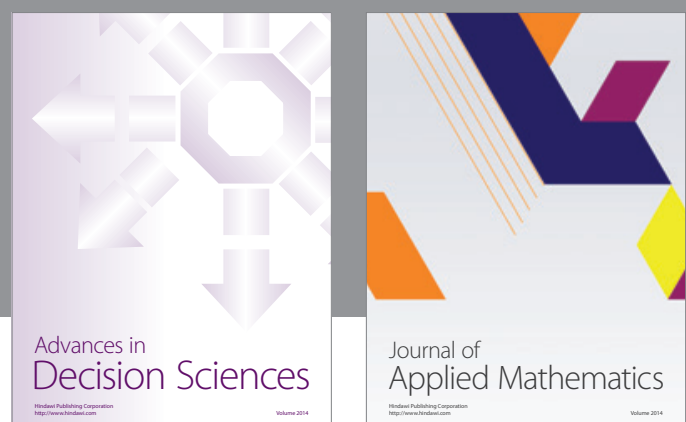

Journal of

Applied Mathematics
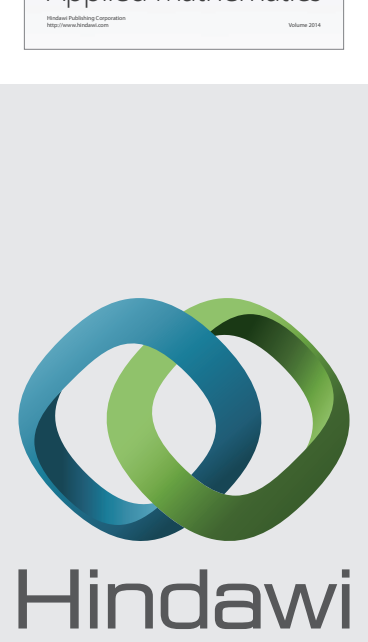

Submit your manuscripts at http://www.hindawi.com
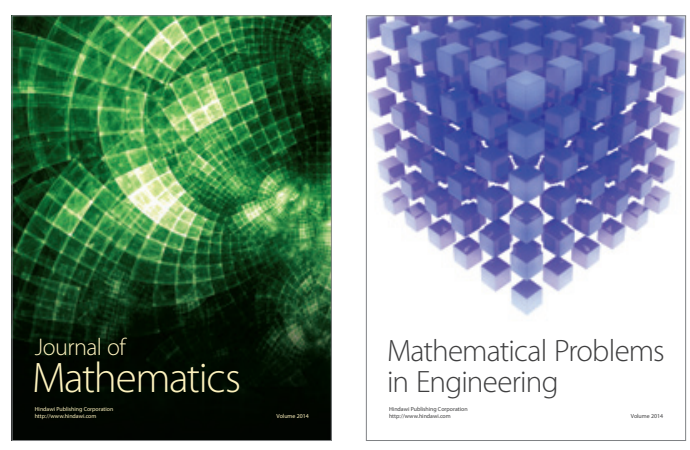

Mathematical Problems in Engineering
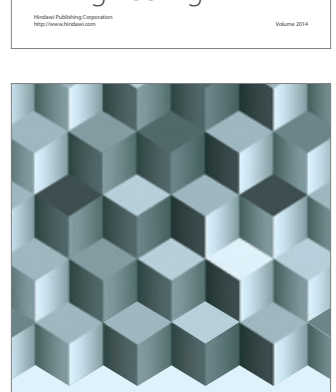

Journal of

Function Spaces
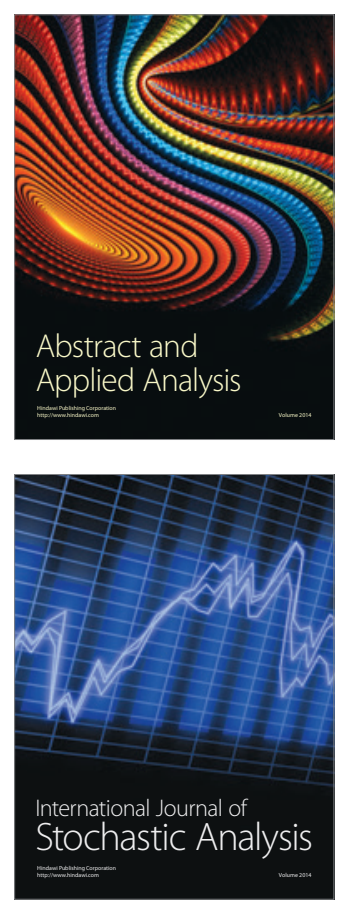

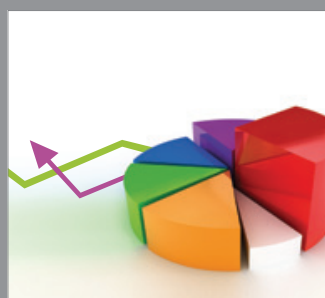

ournal of

Probability and Statistics

Promensencen
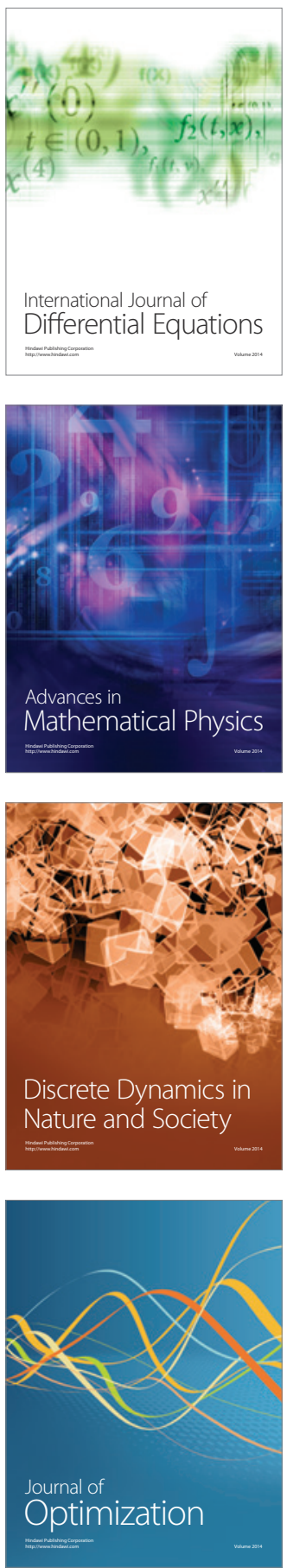